\title{
Single-Server Queue with Markov-Dependent Inter-Arrival and Service Times
}

\author{
I.J.B.F. Adan • V.G. Kulkarni
}

(C) Springer Science + Business Media, LLC 2006

The publisher regrets the following errors which occurred in Queueing Systems, Volume 45, Number 2, October 2003:

- Page 116, line 5: the cross-correlation between $A_{n}$ and $S_{n}$ should read

$$
\rho\left(A_{n}, S_{n}\right)=\rho\left(A_{1}, S_{1}\right)=\frac{\sum_{i=1}^{N} \pi_{i}\left(\lambda_{i}^{-1}-\lambda^{-1}\right)\left(y_{i}-y\right)}{\left\{\left(\sum_{i=1}^{N} 2 \pi_{i} \lambda_{i}^{-2}-\lambda^{-2}\right)\left(\sum_{i=1}^{N} \pi_{i} s_{i}^{2}-\gamma^{2}\right)\right\}^{1 / 2}},
$$

and, hence, the cross-correlation between the inter-arrival time and service time should be +0.17 (instead of +1$)$ in Example 1 and -0.17 (instead of -1 ) in Example 2 in Section 5.

- Page 118, line 9 from bottom: equation (4) should read equation (8)

- Page 119, line 10: $b \Lambda^{-1} e$ at right-hand side should read $v \Lambda^{-1} e$

- Page 130, line 6-8 from bottom: derivation of (A.2) should read

$$
\begin{aligned}
\left|u \tilde{G}_{i}(s) p_{i, i}+\frac{s}{\lambda_{i}}-1\right| & \geq\left|\frac{s}{\lambda_{i}}-1\right|-u \tilde{G}_{i}(0) p_{i, i} \geq 1+\frac{\delta}{\lambda_{i}}-u \tilde{G}_{i}(0) p_{i, i} \\
& >u \tilde{G}_{i}(0)-u \tilde{G}_{i}(0) p_{i, i}=\sum_{j \neq i} u \tilde{G}_{i}(0) p_{i, j} \\
& \geq \sum_{j \neq i}\left|u \tilde{G}_{i}(s) p_{i, j}\right| .
\end{aligned}
$$

- Page 133, reference [28]: (1964) should read (1991) 